\title{
MicroRNA-29a promotes apoptosis of monocytes by targeting STAT3 during sepsis
}

\author{
X. Song ${ }^{1}$, C.T. Wang ${ }^{1}$ and X.H. Geng ${ }^{2}$ \\ ${ }^{1}$ Department of ICU, Shengli Hospital of Shandong University, Shandong, China \\ 2Department of ICU, Dong E People's Hospital, Shandong, China
}

Corresponding author: C.T. Wang

E-mail:w_chunting@163.com

Genet. Mol. Res. 14 (4): 13746-13753 (2015)

Received May 26, 2015

Accepted August 19, 2015

Published October 28, 2015

DOI http://dx.doi.org/10.4238/2015.October.28.37

ABSTRACT. Sepsis is a major cause of morbidity and mortality in critically ill patients. The sepsis syndrome results from a dysregulated inflammatory response to infection that leads to multiple-organ failure, but the underlying mechanisms remain poorly understood. More and more reports show that microRNAs (miRNAs) play an important role in sepsis. In the progression of this syndrome, cells change their behavior in response to cytokines stimulated by sepsis, such as interleukin-10 (IL-10). IL-10 can activate JAK2-STAT3 in the cells to protect them from damage. miR-29a is a potential miRNA directly targeting STAT3. In this study, we investigate the role of miR-29a in targeting STAT3 during sepsis. When cells were treated with IL-10, STAT3 was activated in monocytes, as determined using western blotting. It was verified that STAT3 was a new target gene of miR-29a. miR-29a could inhibit IL-10-induced cytokine release by targeting JAK-STAT3 in monocytes. In conclusion, this study demonstrates for the first time that miR-29a inhibits STAT3 in human monocytes during sepsis.

Key words: miR-29a; Monocytes; Sepsis; STAT3 


\section{INTRODUCTION}

Sepsis is a major cause of morbidity and mortality in critically ill patients. The sepsis syndrome results from a dysregulated inflammatory response to infection that leads to multipleorgan failure (López-Abarrategui et al., 2013; Liesenfeld et al., 2014). A common and severe complication of sepsis is renal insufficiency. Impaired renal function accentuates the pathogenesis of sepsis through the loss of metabolic, fluid, and electrolyte homeostasis (Hotchkiss et al., 2013; Florescu and Kalil, 2014), and the development of kidney dysfunction doubles the risk for mortality in septic patients (Boomer et al., 2014). Bacterial sepsis and acute endotoxemia induce a number of defects, but the underlying mechanisms remain poorly understood.

MicroRNAs (miRNAs) are a class of RNA molecules that control posttranscriptional gene expression primarily by complementary base pairing with specific "seed" sequences in the 3' -untranslated region (3'-UTR) of their target mRNAs (Vandooren et al., 2013). The expression levels of specific miRNAs can have diagnostic value for various forms of malignancy (Ivády et al., 2011; Sinkovics, 2012) and may also provide insight into disease pathogenesis (Hennessy et al., 2010; Reid et al., 2011). Recently, a number of studies have looked at a limited set of miRNAs and have shown that their expression is altered in the context of inflammation or sepsis, both in vitro and in vivo (Ma et al., 2013; Wang et al., 2013; Wang et al., 2014). Reports have shown that miR187, miR-146a, etc., play important roles in sepsis.

In the current study, we examined the role of miR-29a in the regulation of interleukin-10 (IL-10)-induced STAT3 in the context of inflammation and determined the molecular basis for lipopolysaccharide (LPS) hyporesponsiveness and STAT3 expression in the presence of IL-10 in human monocytes. This study demonstrates, for the first time, that miR-29a inhibits STAT3 expression and plays important roles in human monocytes during sepsis.

\section{MATERIAL AND METHODS}

\section{Cell culture}

THP-1 cells were purchased from the ATCC (Manassas, VA, USA). The cells were maintained in Dulbecco's modified Eagle's medium (Gibco, USA) supplemented with 10\% fetal bovine serum in a humidified incubator at $37^{\circ} \mathrm{C}$ and $5 \% \mathrm{CO}_{2}$. Monocytes were isolated from the blood of mice in a mouse model of sepsis or from healthy mice.

\section{ELISA}

Cells were grown overnight on 12 -well plates $\left(4 \times 10^{5}\right.$ cells/well), supernatants were collected, and cytokines were determined by a sandwich enzyme-linked immunosorbent assay (ELISA) according to the manufacturer directions (R\&D Systems). Absorption was measured at $450 \mathrm{~nm}$ on a Versa Max microplate reader using SoftMaxPro software 5.2 (Molecular Devices, Sunnyvale, CA).

\section{Generation of luciferase reporter constructs}

An approximately 2-kb upstream regulatory region from the major transcription start site of the STAT3 gene was amplified by PCR from human genomic DNA and cloned into pGL4-Basic 
(Promega, WI, USA) by using Nhel and HindIII, generating the STAT3-luciferase reporter plasmid. A series of different 5'-truncated fragments were created by PCR amplification from the 2-kb-reporter plasmid and inserted into the pGL4-Basic vector as described above. Site-directed mutagenesis was performed with a MutanBEST kit (Takara, Japan), using the reporter plasmid as a template. The site-specific mutations were confirmed by sequencing.

\section{Lentivirus vectors and lentivirus production}

The pLKO.1-puro-miR-29a vector was created by annealing of sense (5'-ccggcttccagtcga ggatgtttacactcgagtgtaaacatcctcgactggaagttttg-3') and antisense (5'-aattcaaaaacttccagtcgaggatgt ttacactcgagtgtaaacatcctcgactggaag-3') oligonucleotides to each other and subsequent cloning of the resulting double-stranded DNA molecule into the Agel/EcoRI sites of the pLKO.1-puro vector (kindly provided by Sheila Stewart). The empty pLKO.1-puro vector was used as a control. Lentiviruses were produced by transfecting packaging cells (human embryonic kidney 293T cells) with a 3-plasmid system.

\section{Transfection and dual-luciferase assay}

Cells were plated on 24-well plates $24 \mathrm{~h}$ before transfection. Transient transfection was carried out in Opti-MEM medium by using Lipofectamine-2000 according to the manufacturer protocol (Invitrogen, CA, USA). About $6 \mathrm{~h}$ later, the medium was changed, and the cells incubated at $37^{\circ} \mathrm{C}$ for the indicated time. Cells were washed once with phosphate-buffered saline (PBS), and luciferase assays were performed using a dual-luciferase assay system (Promega) according to the supplier protocol. Readings were taken with a Lumat LB 9507 instrument (Berthold Technologies, Germany).

\section{RNA isolation and real-time RT-PCR}

Total RNA was isolated from the cells by using Trizol reagent (Invitrogen) following the manufacturer instructions. Briefly, the cells were lysed in Trizol and then mixed with chloroform. The lysate was centrifuged to separate RNA, DNA, and protein, and total RNA was recovered, precipitated with isopropanol, and washed in $75 \%$ ethanol to remove impurities before being dissolved in water. After that, $2 \mu \mathrm{g}$ of RNA was taken and treated with DNase to remove contaminating DNA prior to reverse transcription (RT) to cDNA by using a SYBR ${ }^{\circledR}$ PCR kit (Takara). To measure mRNAexpression, real-time RT-PCR was performed using a sequence detector (ABIPrism, Applied Biosystems, USA). The relative expression levels were calculated by comparing Ct values of the samples with those of the reference, and all data were normalized to the internal control, glyceraldehyde-3-phosphate dehydrogenase.

\section{Western blotting}

The cells were scraped from the dishes, cellular protein extracts prepared by homogenization in an ice-cold lysis buffer, their lysates obtained by centrifugation at 12,000 $\mathrm{g}$ for $20 \mathrm{~min}$, and the total protein concentration determined using the Lowry method. Equal amounts of protein, separated by SDS-PAGE, were electrophoretically transferred to a polyvinylidene difluoride membrane at $320 \mathrm{~mA}$ for $2 \mathrm{~h}$ at a low temperature, and the membrane was blocked with $5 \%$ fatfree milk with $0.05 \%$ Tween 20 in PBS (PBST). Subsequently, the membrane was probed with the 
primary antibodies. The blots were washed in PBST and then incubated in anti-mouse lgG or antirabbit IgG secondary antibody for about $3 \mathrm{~h}$ at room temperature. The blots were washed in PBST (3X, $10 \mathrm{~min}$ each), and the proteins were finally visualized using enhanced chemiluminescence based on the manufacturer instructions.

\section{Statistical analysis}

Each experiment was repeated at least three times, and Student t-tests were performed to determine the statistical significance for the assays of promoter activity, migration, fluorescenceactivated cell sorting, and colony formation. Data are reported as means \pm standard errors.

\section{RESULTS}

\section{Sepsis induces IL-10 expression in human monocytes}

There are many inflammatory cytokines secreted during sepsis which play important roles in the progression of the disease, and IL-10 is one of them. To investigate whether IL-10 expression increases during sepsis, monocytes were isolated from humans with sepsis or from normal controls, and the ELISA results showed that the IL-10 level was increased in the monocytes from 10 patients with sepsis (Figure 1A). The expression of IL-10 was confirmed for THP-1 cells with LPS treatment by using ELISA (Figure 1B).

\section{A}

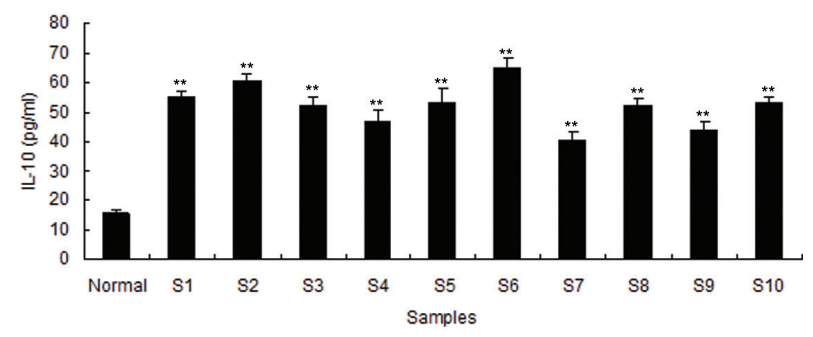

B

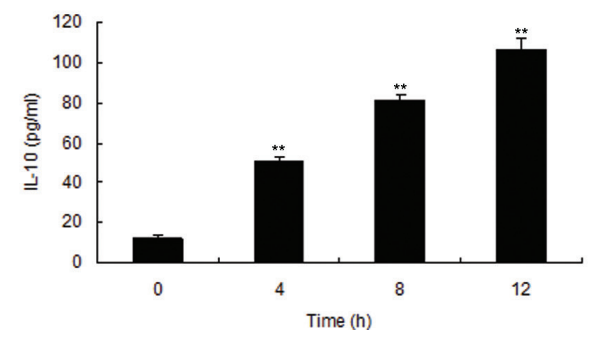

Figure 1. IL-10 expression in monocytes is increased during sepsis. A. Fresh monocytes were isolated from humans with sepsis, and supernatants were collected. IL-10 was analyzed by ELISA. B. IL-10 expression in THP-1 monocytes with LPS treatment was confirmed by ELISA. Cells were stimulated with LPS $(20 \mathrm{nM})$, and the growth medium was collected for ELISA. ${ }^{* *} \mathrm{P}<0.01$. 


\section{IL-10 induces inhibition of apoptosis in monocytes by STAT3}

To determine the role of IL-10 in the regulation of cell apoptosis and proliferation, THP-1 cells were exposed to IL-10 with or without LPS. It was found that IL-10 promoted cell proliferation and protected cells from apoptosis (Figure $2 \mathrm{~A}$ and B). Cytokines such as IL-6 can activate the JAKSTAT3 signaling pathway, and we wanted to know whether IL-10 can stimulate STAT3 activation. Fresh monocytes and THP-1 cells were treated with IL-10, and the data from western blotting showed that the p-STAT3 level increased in a time-dependent manner (Figure 2C).

A

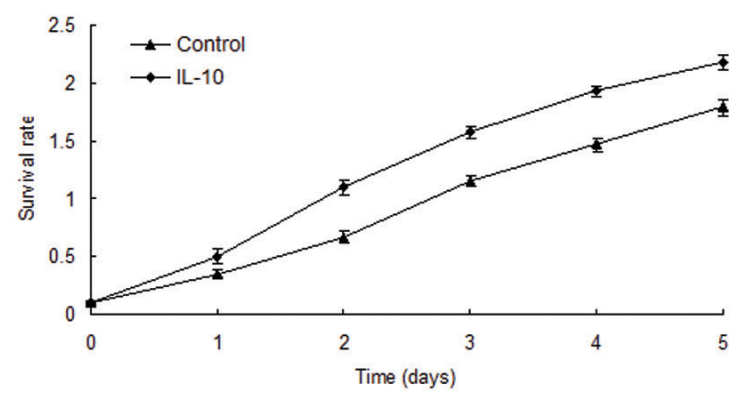

$\mathrm{B}$
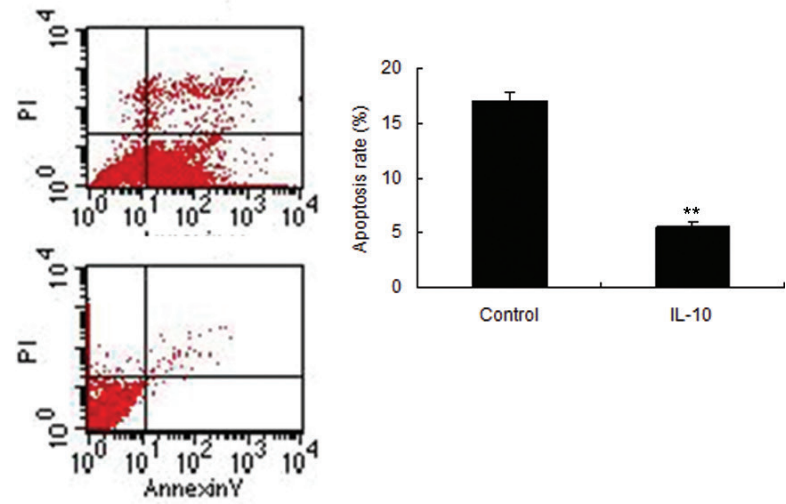

C

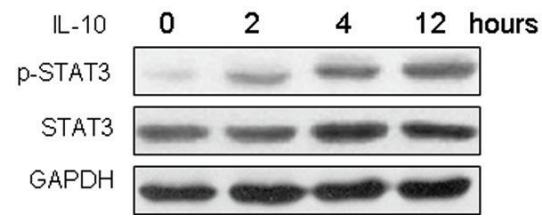

Figure 2. IL-10 promotes cell proliferation and inhibits cell apoptosis of human monocytes by STAT3. A. Monocytes were treated with or without IL-10, and cell proliferation was assayed by the MTT method. B. Monocytes were treated with or without IL-10, and cell apoptosis was assayed by flow cytometry. C. Freshly isolated monocytes were stimulated with IL-10 (10 nM) for the indicated times, and proteins were isolated for western blotting with STAT3 staining. ${ }^{* *} \mathrm{P}<0.01$. 


\section{STAT3 is a new target gene of miR-29a in monocytes}

We then investigated the mechanisms by which miRNAs regulate STAT3 expression. Bioinformatic analysis showed that miR-29a may be a potential miRNA targeting STAT3 (Figure 3A). Thus, a luciferase reporter plasmid with the 3'-UTR of STAT3 was constructed. The luciferase activity of the wild-type luciferase reporter plasmid (STAT3-WT) was much lower in THP-1 cells than the activity of the luciferase reporter plasmid with the 3'-UTR of STAT3, and the luciferase activity of STAT3-Mut was rescued in cells (Figure 3B). We next examined whether miR-29a could regulate endogenous STAT3 expression in monocytes. Compared with the controls, endogenous STAT3 mRNA levels (Figure 3C) and protein levels (Figure 3D) were down-regulated when cells were transfected with miR-29a. These data indicate that STAT3 is a direct target gene of miR-29a.

A

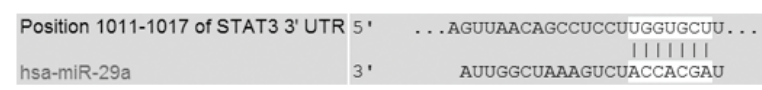

C

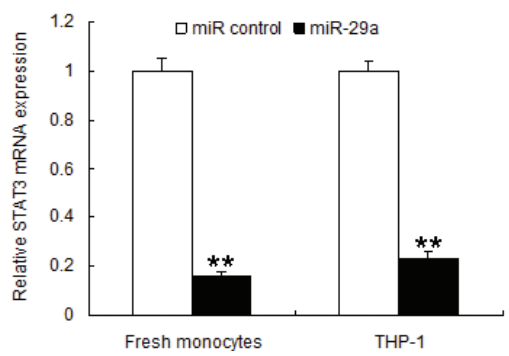

B

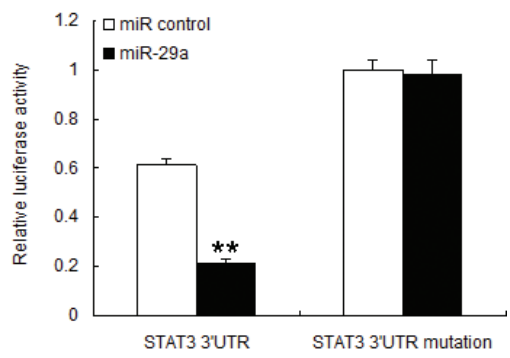

Figure 3. miR-29a down-regulates STAT3 expression in THP-1 cells. A. The 3'-UTR of the STAT3 gene contains binding sites for miR-29a, according to bioinformatic analysis. B. miR-29a suppressed the expression of a luciferase reporter gene harboring the 3'-UTR of the STAT3 gene. Cells were transiently cotransfected with a negative control (mock) or miR-29a, together with the indicated luciferase constructs, and luciferase activity was analyzed $48 \mathrm{~h}$ later. Data are presented as the relative firefly luciferase activity normalized to the Renilla luciferase activity from the same construct. C. miR-29a restoration down-regulated STAT3 in THP-1 cells and fresh monocytes. Cells were transfected with miR-29a or miR-control for $48 \mathrm{~h}$ and then collected for western blot analysis. D. miR-29a restoration downregulated STAT3 in THP-1 cells and fresh monocytes. The data presented are means and standard deviations of the results from three independent experiments. ${ }^{* *} \mathrm{P}<0.01$.

\section{miR-29a inhibits IL-10-induced cell growth through down-regulation of STAT3}

The data described above showed that miR-29a inhibited STAT3 mRNA and protein expression in monocytes. The remaining question was whether miR-29a inhibits cell proliferation by down-regulation of STAT3. To answer this question, the cells were treated with IL-10 and infected with LV-miR-29a, and the results of flow cytometry indicated that apoptosis increased in the cells with LV-miR-29a (Figure 4A). An MTT assay showed that miR-29a could inhibit cell proliferation (Figure 4B). 
A

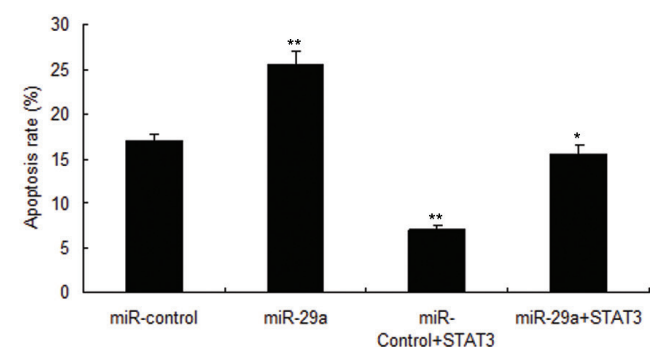

B

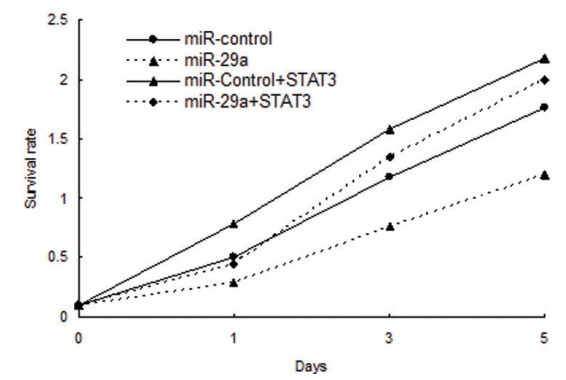

Figure 4. miR-29a promotes cell apoptosis and inhibits cell proliferation. A. Cells were transfected with miR-29a or miR-control, stimulated with IL-10 for $48 \mathrm{~h}$, dyed with fluorescein isothiocyanate-annexin V, and then collected for flow cytometry. B. Cells were transfected with miR-29a or miR-control, stimulated with LPS for 48 and $96 \mathrm{~h}$, and then subjected to MTT assay. ${ }^{* *} \mathrm{P}<0.01 ;{ }^{*} \mathrm{P}<0.05$.

\section{DISCUSSION}

In this study, we investigated the molecular mechanism of STAT3 regulation by miR-29aSTAT3 in monocytes with sepsis. For the first time, we demonstrated that miR-29a inhibits the IL10-STAT3 signaling pathway in monocytes.

Signal transducers and activators of transcription (STATs) are the principle signaling proteins of many cytokines and growth factors in mammals. STATs play a critical role in regulating immune cell homeostasis, differentiation, and cellular functions (Wu et al., 2013; Trilling et al., 2014). There are seven STAT proteins (STAT1, STAT2, STAT3, STAT4, STAT6, and the STAT5a and STAT5b isoforms), each with established roles in immune cell functioning. The key regulation of STATs is mediated by phosphorylation, typically by Janus kinases (JAKs). STAT1 and -2 respond to interferons (IFNs), thereby promoting IFN-stimulated genes and antiviral immunity. STAT3 responds to factors including IL-6, IL-10, and vascular endothelial growth factor and is involved in T-helper cell 17 (Th17) and Treg cell development and tumorigenesis (Lin et al., 2013; Nieminen et al., 2013). STAT4 and STAT6 control Th1 and Th2 cell differentiation in response to IL-12 and IL-4/13, respectively (Fontan and Melnick, 2013; Meisch et al., 2013; Singh et al., 2013). Due to the large involvement of STATs in a variety of cell processes, it is critical that their activity be tightly regulated.

The IL-10 receptor, which is also constitutively expressed on these cells, activates the JAK/STAT pathway, inducing phosphorylation and dimerization of STAT3, which binds to the GAS of the promoter region of target genes (Pattison et al., 2013; Haque et al., 2014). In the current study, we showed that STAT3 is phosphorylated in response to IL-10, demonstrating that this pathway is activated in monocytes. Most importantly, we found that miR-29a regulates the function 
of monocytes by directly activating STAT3.

In summary, we examined the role of miR-29a in sepsis. STAT3 was predicted as the target gene of miR-29a by TargetScan and verified the predicted result. This study demonstrates that miR-29a inhibits sepsis by inhibition of STAT3 stimulated by IL-10 in human monocytes.

\section{Conflicts of interest}

The authors declare no conflict of interest.

\section{REFERENCES}

Boomer JS, Green JM and Hotchkiss RS (2014). The changing immune system in sepsis: is individualized immuno-modulatory therapy the answer? Virulence 5: 45-56.

Florescu DF and Kalil AC (2014). The complex link between influenza and severe sepsis. Virulence 5: 137-142.

Fontan L and Melnick A (2013). Discovering what makes STAT signaling TYK in T-ALL. Cancer Discov. 3: 494-496.

Haque A, Koide N, Odkhuu E, Tsolmongyn B, et al. (2014). Mouse pyrin and HIN domain family member 1 (pyhin1) protein positively regulates LPS-induced IFN- $\beta$ and NO production in macrophages. Innate Immun. 20: 40-48.

Hennessy EJ, Parker AE and O'Neill LA (2010). Targeting Toll-like receptors: emerging therapeutics? Nat. Rev. Drug Discov. 9: 293-307.

Hotchkiss RS, Monneret G and Payen D (2013). Sepsis-induced immunosuppression: from cellular dysfunctions to immunotherapy. Nat. Rev. Immunol. 13: 862-874.

Ivády B, Béres BJ and Szabó D (2011). Recent advances in sepsis research: novel biomarkers and therapeutic targets. Curr. Med. Chem. 18: 3211-3225.

Liesenfeld O, Lehman L, Hunfeld KP and Kost G (2014). Molecular diagnosis of sepsis: new aspects and recent developments. Eur. J. Microbiol. Immunol. 4: 1-25.

Lin L, Hou J, Ma F, Wang P, et al. (2013). Type I IFN inhibits innate IL-10 production in macrophages through histone deacetylase 11 by downregulating microRNA-145. J. Immunol. 191: 3896-3904.

López-Abarrategui C, Del Monte-Martínez A, Reyes-Acosta O, Franco OL, et al. (2013). LPS inmobilization on porous and nonporous supports as an approach for the isolation of anti-LPS host-defense peptides. Front. Microbiol. 4: 389.

Ma Y, Vilanova D, Atalar K, Delfour O, et al. (2013). Genome-wide sequencing of cellular microRNAs identifies a combinatorial expression signature diagnostic of sepsis. PLoS One 8: e75918.

Meisch JP, Vogel RM, Schlatzer DM, Li X, et al. (2013). Human $\beta$-defensin 3 induces STAT1 phosphorylation, tyrosine phosphatase activity, and cytokine synthesis in T cells. J. Leukoc. Biol. 94: 459-471.

Nieminen JK, Niemi M, Sipponen T, Salo HM, et al. (2013). Dendritic cells from Crohn's disease patients show aberrant STAT1 and STAT3 signaling. PLoS One 8: e70738.

Pattison MJ, MacKenzie KF, Elcombe SE and Arthur JS (2013). IFN $\beta$ autocrine feedback is required to sustain TLR induced production of MCP-1 in macrophages. FEBS Lett. 587: 1496-1503.

Reid G, Kirschner MB and van Zandwijk N. (2011). Circulating microRNAs: association with disease and potential use as biomarkers. Crit. Rev. Oncol. Hematol. 80: 193-208.

Singh UP, Singh NP, Guan H, Busbee B, et al. (2013). Leptin antagonist ameliorates chronic colitis in IL-10\% mice. Immunobiology 218: 1439-1451.

Sinkovics JG (2012). Molecular biology of oncogenic inflammatory processes. I. Non-oncogenic and oncogenic pathogens, intrinsic inflammatory reactions without pathogens, and microRNA/DNA interactions. Int. J. Oncol. 40: 305-349.

Trilling M, Le VT, Rashidi-Alavijeh J, Katschinski B, et al. (2014). "Activated" STAT proteins: a paradoxical consequence of inhibited JAK-STAT signaling in cytomegalovirus-infected cells. J. Immunol. 192: 447-458.

Vandooren J, Van den Steen PE and Opdenakker G (2013). Biochemistry and molecular biology of gelatinase B or matrix metalloproteinase-9 (MMP-9): the next decade. Crit. Rev. Biochem. Mol. Biol. 48: 222-272.

Wang HJ, Deng J, Wang JY, Zhang PJ, et al. (2014). Serum miR-122 levels are related to coagulation disorders in sepsis patients. Clin. Chem. Lab. Med. 52: 927-933.

Wang ZH, Liang YB, Tang H, Chen ZB, et al. (2013). Dexamethasone down-regulates the expression of microRNA-155 in the livers of septic mice. PLoS One 8: e80547.

Wu Y, Yang L, Mei X and Yu Y (2013). Selective inhibition of STAT1 reduces spinal cord injury in mice. Neurosci. Lett. 580: 7-11. 